\title{
Erratum to: Rare abdominal metastases from occult lobular breast cancer: report of two cases
}

Francesco Razzetta $\cdot$ Elisabetta Tassara $\cdot$

Francesca Saro • Maria Sironi · Giovanni D’Ambrosio

Published online: 14 July 2011

(C) Springer-Verlag 2011

Erratum to: Updates Surg (2011) 63:129-133

DOI 10.1007/s13304-011-0047-x

The names and affiliations of four of the five authors were inadvertently omitted. Their details are now supplied here.

Francesco Razzetta - Elisabetta Tassara - Francesca Saro · Maria Sironi · Giovanni D’ Ambrosio

F. Saro $\cdot$ M. Sironi $(\bowtie)$

S.C. Anatomia e Istologia Patologica, P.O. di Sestri Levante, V. Terzi 43/A, 16039 Sestri Levante, Italy e-mail: msironi@asl4.liguria.it

F. Razzetta $\cdot$ E. Tassara · G. D'Ambrosio

S.C. Chirurgia, P.O. di Lavagna, Lavagna, Italy

The online version of the original article can be found under doi:10.1007/s13304-011-0047-x.

F. Saro $\cdot$ M. Sironi $(\bowtie)$

S.C. Anatomia e Istologia Patologica,

P.O. di Sestri Levante, V. Terzi 43/A,

16039 Sestri Levante, Italy

e-mail: msironi@asl4.liguria.it

F. Razzetta - E. Tassara - G. D’Ambrosio

S.C. Chirurgia, P.O. di Lavagna, Lavagna, Italy 